\title{
Quantitative assessment of sad emotion
}

\author{
Nicoladie D. Tam \\ Department of Biological Sciences, University of North Texas, Denton, USA
}

\section{Email address:}

nicoladie.tam@unt.edu

\section{To cite this article:}

Nicoladie D. Tam. Quantitative Assessment of Sad Emotion. Psychology and Behavioral Sciences. Vol. 4, No. 2, 2015, pp. 36-43. doi: $10.11648 /$ j.pbs.20150402.11

\begin{abstract}
Based on the disparity theory of emotion, the role of sad emotion is an internal assessment of the error-correction process to reduce the disparity between the expected and actual outcomes (loss reduction) in the reality check process. This computational theory of emotion is consistent with the psychological characteristics that sadness is an emotional response to the sense of loss (such as loss of loved ones, valuables, possessions, or achieved goals). This emotional theory of sadness also includes the emotional resolution process by accepting that nothing can be done to change the actual outcomes, and resolving the emotion by reducing the perceived loss. This self-corrective mechanism is used as an internal feedback to assess the incongruence between the expectation and the actuality, such that the perceived loss can be reduced, resolving the sad emotion in the process. Thus, sadness can serve as a motivating feedback to an individual to make a decision to reduce the loss in the emotional resolution process. The classical ultimatum game (UG) paradigm is used to elicit self-generated emotion in human subjects experimentally in response to the disparity between the proportions of money being offered to share with. Results showed that the sadness level is quantified by the sadness stimulus-response function. The level of sadness intensity is proportional to perceived loss (or inversely proportional to the perceived gain). The results also showed that there was a shifting of the baseline sadness level from a less sad level for the acceptance decision to a more sad level for the rejection decision. This shows that the sad emotion can be resolved by accepting the monetary offer in the UG paradigm, which reduces the loss compared to the decision to reject the money. These results confirmed the emotional disparity hypothesis that the level of sadness is proportional to the perceived loss, and sadness can be resolved by reducing the loss in the self-regulated internal processing of emotion. Implications on emotional intelligence are also addressed so that one of the effective skills to resolve sadness is the reduction of the perceived losses.
\end{abstract}

Keywords: Emotion, Sadness, Fairness, Ultimatum Game, Decision Making, Loss Reduction, Emotional Intelligence

\section{Introduction}

Toward the goal of quantifying the role of emotions based on the theory of emotional processing for self-discovered error-detection and error-correction [1,2], previous studies [3-6] sddsg [3-5] had demonstrated that emotional intensity is proportional to the perceived gain/loss signals. According to this emotional disparity theory, the hypothesis is that intensity of emotion is proportional to the disparity between what a person wants (the expectancy) and gets (the actuality).

According to this emotional disparity theory based on the EMOTION-I model [1] and EMOTION-II model [2], happiness is an emotional feedback that indicates a congruence with the expectancy and actuality, while unhappiness indicates an incongruence between the expectancy and the actuality. The greater the congruency, the greater the happiness emotional intensity is. The greater the incongruency, the greater the unhappy emotional intensity is.

In simple intuitive terms, happiness is a measure of what is right, and unhappiness is a measure of what is wrong. This emotional disparity theory is consistent with the intuition that we often ask a person who is feeling unhappy/sad, "What is wrong?" Thus, sad emotion is a feedback to a person that something is wrong (undesirable) with their expectation of what happened (i.e., something had happened that he/she does not want) - an indication of incongruency. On the other hand, if the expectation is exactly the same as what had happened (congruent with the desirable), then the person would feel happiness - an indication of congruency.

It is well recognized in psychology that sadness is an emotional response to the sense of loss. The loss can be anything that is valuable to a person, such as the loss of a loved one, the loss of possession, or the loss of achieved goals. Sadness occurs when a person perceives a sense of 
loss to something that is valuable that he/she cannot change the outcome. Given this intuitive definition of sadness, this paper will derive the theoretical foundation according to the emotional disparity theory. The computational role of sad emotion is to assess the losses by comparing the difference between the expectancy (predicted outcomes) and the reality (actual outcomes) in the reality check process.

\subsection{Theory of Emotional Disparity in Assessing Reality}

According to this theory of emotional disparity, emotional processing is a series of neurobiological mechanisms for selfdiscovery of errors and self-correction of errors. The theory is derived based on the evolutionary process for survival, in which the survivability of an individual (humans or animals) increases when the internal brain model of the external world is an accurate model. That is, in order to survive in the real world, the brain has to create a model of the external world to predict its outcomes, so that it can respond to the environment appropriately. According to this emotional theory, emotion is a feedback that indicates the discrepancy between the expected (predicted) and the actual outcomes. The emotional resolution process is an error-correction process to reduce the disparity — an error-minimization process computationally.

This emotional response serves as an internal feedback for an individual to assess the accuracy of the expectancy (or prediction) of the real world outcomes for survival. When the internal prediction (by the brain) is an accurate representation of the external world, the survivability increases. There is a match between the expected and actual outcomes. No corrections are needed to correct the internal (brain) model. This represents the contented state of happiness, where no actions are needed to correct anything.

When there is a discrepancy between the expectancy and the actual outcomes, the mismatch in expectation creates an incongruency, triggering an unhappy emotion as a feedback indicating something is wrong. In order to resolve the emotional discourse, such disparity has to be reconciled. Thus, the unhappy emotion serves as an internal indicator to an individual that something is wrong in he/her expectation, which does not match the reality.

\subsection{Initial Surprise (Shock) Phase in Error-Detection}

This initial recognition stage of disparity is often responded by a surprise (or a shock) response. Although this initial error-detection recognition process is often an autonomic process that may not reach the cognitive level of awareness, it provides an internal (subconscious) assessment/detection of the discrepancy between what a person wants and gets. According to this emotional disparity theory, the initial stage is the surprise (shock) phase when the disparity is first detected - the error-detection phase.

Once this error (disparity) condition has been detected by the autonomic system, the next stage of emotional processing is to resolve the emotion by making (subconscious) internal neurological attempts to correct any incongruency between the expectation and the actuality. Different unhappy emotions (such as angry, sad and fear) represent different stages of emotional processing to resolve the unhappy emotion, so that a congruency can be achieved eventually to reach the happy/contented state.

\subsection{Anger Stage of Emotional Processing to Resolve Unhappy Emotion}

Once the initial surprise phase has passed, the next phase in emotional processing is to identify where the source of error comes from, in order to correct for the incongruency. The source of error may come from the input (sensory/perceptual error), the output (execution error), or the internal prediction/modeling error (belief system error). Since an individual is often unaware of these internal errors (hidden errors), it is often easier to assume that the source of error comes from an external source rather than an internal source. If the source of error is identified (or assumed - in a resolution strategy without identifying it first), then an attempt would be made to resolve the disparity accordingly.

There are two ways to correct the disparity: change the world or change oneself. If the source of error is assumed to be coming from the external source (i.e., if the outside world is assumed to have caused the disparity in expectation), then an attempt would be made to change the world (instead of changing oneself). When such an attempt to change the world fails, an angry emotion is felt as a feedback to the individual that something is wrong. That is, if the attempt were successful, it would not elicit an anger response.

Anger serves as a feedback indicating a failed/futile attempt had been made to reduce the disparity. Furthermore, anger also indicates that the person could not accept the fact that nothing can be done to change the actual outcomes (when it had assumed that the source of disparity came from the external source). If the person had accepted that nothing could be done to change the world, it would evoke sadness rather than anger. It is only when an attempt to change the world had failed and the person cannot accept the outcomes, then the angry emotion sets in. Thus, according to this emotional disparity theory, the anger emotion serves as an internal feedback to indicate a failed attempt had been made to change the reality, but it is unacceptable to admit failure.

\subsection{Sadness Stage of Emotional Processing to Resolve Unhappy Emotion}

After the anger stage, the next stage in the emotional processing is to accept the fact that nothing can be done to correct the disparity in expectation by changing the world. During this acceptance phase, when the individual has not found an alternate solution to correct the disparity yet, then the sad emotion would set in. Thus, sadness is an emotional feedback that indicates the intermediate stage of errorcorrection, in which an appropriate solution has not been found yet to reduce the disparity, while accepting the fact that the emotion cannot be resolved by changing the reality (or blaming the world for its erroneous expectation). The 
unhappy emotion will not be resolved until an effective solution is found to identify and correct the source of error. The source of error can stem from the input (faulty perception), the output (faulty execution), or the expectation (faulty belief). It is the recognition of the sense of loss (that failed to meet the expectation) that triggers the sad emotion.

\subsection{Proportionality Hypothesis between Emotional Intensity and Disparity}

Based on this emotional disparity theory, the unhappy emotional intensity is proportional to the amount of disparity between the expectancy and the actuality. The greater the degree of discrepancy, the greater the emotional intensity is. Given this quantifiable measure of emotional intensity in relation to the disparity between what a person wants and gets, this emotional disparity hypothesis can be tested and validated experimentally in human subjects without resorting to using any traditional subjective stimuli commonly used in most psychological studies of emotions.

If a person gets what he/she wants, this represents a gain signal. If a person does not get what he/she wants, this represents a loss signal. Thus, the gain/loss signals can be used as a measure to determine the difference between the expectancy and the actuality quantitatively. This provides a powerful quantitative tool to study emotions using numerical measures, without using any of the traditional subjective stimuli, such as facial expressions [7-9], emotional words [10-12] or sad movies [13], as the stimuli for eliciting the sad responses.

Using this quantitative approach to elicit emotional responses, previous studies had addressed the happiness [4, 5], anger [3] and jealousy emotional responses [6]. These studies showed that the intensity of happy and unhappy emotions is related to the disparity between what a person wants and gets proportionally. This paper will focus on the assessment of sad emotional response to the same set of quantifiable stimuli using the Ultimatum Game (UG) as previously described [14-17].

\subsection{Fear as an Emotional Feedback to Identify and Predict the Undesirable Outcomes}

For completeness in the emotional disparity theory, fear is an emotional feedback that predicts the undesirable outcomes, which usually threatens the survival of an individual. Thus, unhappy emotions estimate the survivability by determining whether such estimate is desirable or undesirable relative to survival. If the estimate predicts an increase in survival, it is desirable. If the estimate predicts a decrease in survival, it is undesirable.

Fear is an internal estimate that predicts a decrease in survivability. It is a prediction of the potential danger that threatens the survival. Therefore, even though the prediction by fear is accurate, it is undesirable, so the appropriate response to increase the survivability is to reverse the course of action, so that the anticipated danger can be avoided.

Computationally, this introduces an additional "desirability" factor in the error-correction phase, such that if the prediction is desirable, then the error-recovery process in emotional processing is to reduce the discrepancy between the expected and the actual outcomes. On the other hand, if the prediction is undesirable, then the error-recovery process in emotional processing is to reverse the course of action, so that the undesirable prediction can be averted. That is, instead of minimizing the discrepancy in prediction, the undesirability factor produces a sign-change mathematically, so that the course of action is to maximize the discrepancy between the predicted doom and the actuality in fear emotion processing.

\subsection{Quantifiable Stimuli for Eliciting Emotional Responses Using the Ultimatum Game Experimental Paradigm}

In order to test the emotional disparity hypothesis between the gain/loss signals, the classical UG paradigm in behavioral economics [14-17] can provide an objective, quantifiable stimulus to elicit emotional responses. The resulting emotions elicited can be assessed with respect to perceived gains and losses in money and fairness using the UG experimental paradigm. The UG paradigm is a split-themoney game to share an amount of money between two players (a proposer and a responder). If the responder accepts the monetary offer, both keep the money. If the responder rejects it, both lose the money. This provides the experimental paradigm in which the stimulus variable is the offer-ratio - which can be varied between the proportions of money to be shared by the two players.

In order to ensure that only objective quantifiable stimulus is used to elicit an emotional response, the UG experiment is conducted without any human interactions (by using computer presentation of the monetary offer) or subjective influence by the human experimenter (without using any facial expression of the proposer). This provides the experimental condition in which the only variable varied in the stimulus is the numeric monetary offer-ratio to be shared.

Under these conditions, the emotions elicited are selfgenerated by the human subjects, without any subjective biases by the experimenter or skewed by the experimental environment. The emotional responses are dependent on how the subject perceives the gains or losses relative to the disparity in the offer-ratios, which are not influenced by the experimental conditions except for the disparity variable of the monetary offer-ratio.

Numerous studies had used the UG paradigm to address the decision-making process rather than the emotional responses [18-23]. Other UG studies had addressed how the decision can be affected by the emotional manipulations of the experimental conditions [13, 18, 20, 21, 24-31]. Only recently, the relationship between the offer-ratio stimuli and the emotional responses were addressed systematically based on the present emotional disparity theory with respect to the happy, angry and jealous emotions [3-6, 32], fairness [33, 34] and decision $[35,36]$. This paper focuses on addressing the emotional disparity theory in sad emotion by:

(1) quantifying the emotional response using the stimulusresponse function, and

(2) validating the emotional disparity intensity hypothesis. 


\section{Methods}

Healthy human subjects were recruited to play as the responder using the UG paradigm as described above. The monetary offers were presented on a computer screen, using text description without any image of human facial expression in the offer. This ensures that the emotional responses were not elicited by any other subjective facial cues or suggestions, and the only variable in the stimulus is the numeric monetary offer-ratio. Thus, the emotional response of the human subjects would be self-generated based on their perception of disparity in the monetary offer between the proposer and the responder.

The experimental trials were randomized with the monetary offers ranging from $\$ 1$ to $\$ 9$ to the responder. The offers were presented using a one-shot offer trial, i.e., without repeating the same offer twice. This ensures that the stimulus does not introduce any adaptation (sensitization or desensitization) effects due to prior repeated exposure. The random sequence is generated from a pseudo-random number generator, so that all subjects were presented with the same pseudo-random sequence of offer-ratios. This ensures that the results of all subjects can be analyzed similarly in response to the same pseudo-random sequence of offer-ratios.

After the subjects made the decision to accept or reject the offer, they were asked to self-report their emotional responses to that offer (using a numeric rating scale from +5 to -5$)$. The experiment was designed to measure the selfreported rating of emotions in order to assess the perception of emotions rather than the autonomic emotional response. This provides the metrics for addressing the hypothesis of self-assessment of emotional response rather than the autonomic physiological responses in reaction to the stimulus. The study was approved by the University Institutional Review Board.

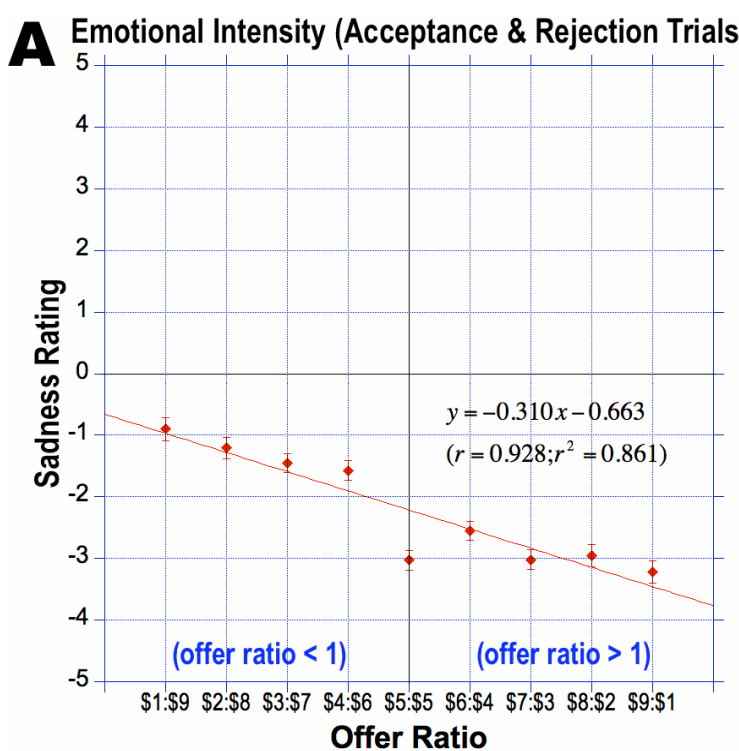

\section{Results}

A total of 230 subjects (age ranging from 18 to 40 ; median age $=21 ;$ male $=85$, female $=145$ ) participated in this study. Fig. 1 shows the self-reported rating of sad emotion for the entire sampled population, independent of whether they accept or reject the offer. Regression analysis using curvefitting for the graph in Fig. 1A shows that sadness intensity is inverse proportional to the offer-ratio (regression function: $y$ $\left.=-0.310 x+0.663 ; r=0.928 ; r^{2}=0.861\right)$. The more inequitable the offer, the greater the intensity of sad emotion was reported, which is consistent with the emotional disparity hypothesis.

\subsection{Proportionality Relationship between Sadness Level and Losses}

As a whole, on average, the subjects reported not sad (all data points are below the $x$-axis) for all offer-ratios, even though they reported a higher level of sadness for the inequitable offers than the hyper-equitable offers proportionally. This shows that even though they are not sad, the subjects are cognizant of the degree of sadness level in proportion to the disparity of the offer shared between the proposer and the responder. Because these ratings are selfreported ratings, they are conscious of their sadness perception by verbalizing it in their self-reported ratings.

It is important to note that the initial sequence of offerratios are randomized during the experiment, but the graphs are plotted in ascending order after the randomized trials are sorted according to the offer-ratios. Thus, the subjects were not cued to the awareness of the increasing disparity of the offer-ratios. The proportionality of the sadness level is revealed only when the data were analyzed according to the subsequent sorted offer-ratios in the regression analysis.

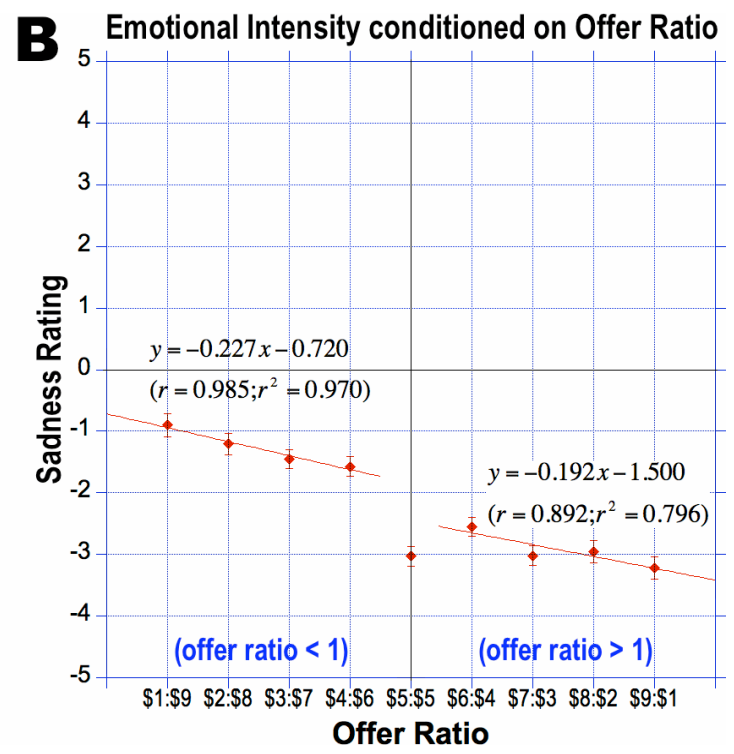

Figure 1. Self-reported sadness rating with respect to monetary offer for the entire population ( $n=230)$. (A) The regression curve-fitting showed an inverse proportional relationship. (B) The regression curve-fitting conditioned on unfair (unfavorable) and hyper-fair (favorable) trials for the same set of data as in (A), which showed different proportionality relationships depending on whether the offers are equitable or not. The error bar represents standard error of mean (SEM). 
Although there is an inversely proportional trend between the sadness level and the offer-ratio, there is a discontinuity at the center of the graph (at the $\$ 5: \$ 5$ equal share offer in Fig. 1). In order to delineate the difference in emotional response to the hyper-equitable offers from the inequitable offers, the graph is fitted with two different regression curvefitting functions (left- and right-half of Fig. 1B) instead of a single regression function (Fig. 1A).

When the emotional responses are separated according to the equitable/inequitable offers, there is a discrete shift in the baseline sadness level (indicated by the $y$-intercept). The $y$ intercepts are different, but the slopes of the stimulusresponse curve are similar. This shows there is slight sudden shift in the baseline sadness level in response to the inequitable offers compared to the hyper-equitable offers, but there is no change in the sadness sensitivity (as indicated by the slope).

At the equal share offer $(\$ 5: \$ 5)$, the sudden shift in sadness level is much more pronounced. This sudden shift is consistent with the discrete shift at the absolute equity offer for other emotions (including happiness [4, 5], anger [3] and jealousy [6]), and for fairness perception also [33, 34]. This shows that when the share is a 50/50 split, the offer is perceived as the fairest for both parties. This resolves the conflict, in which the gain for one party (responder) is a loss for the other party (proposer), if both fairness and monetary

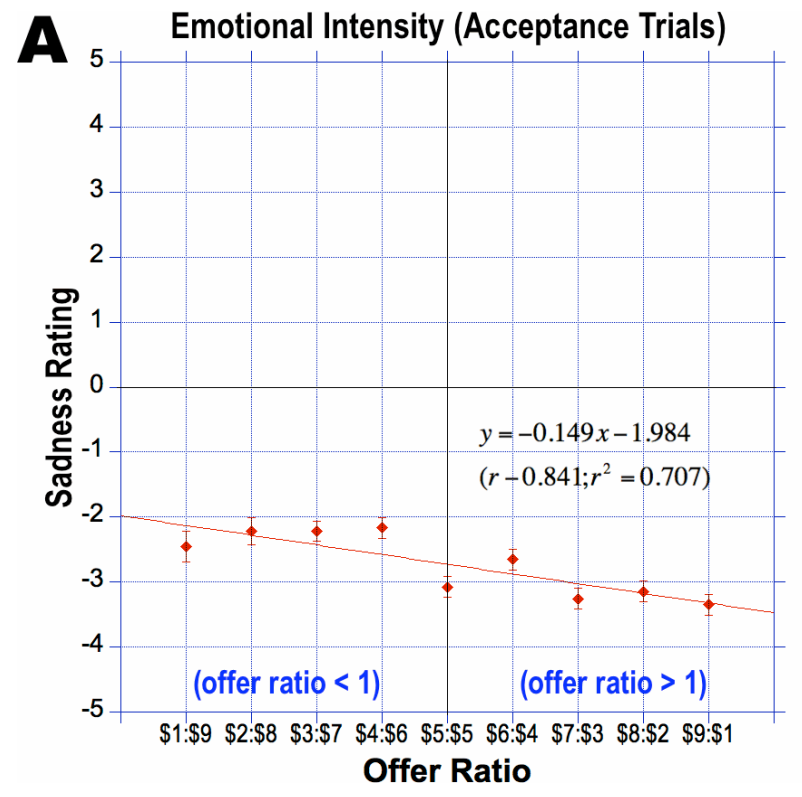

gains are optimized for both parties. That is, if the disparity is to minimize not just for oneself, but also for the other party, then the optimal scenario is the even-split offer where it is fair to both parties. It would be a win-win scenario for monetary gain for both parties. This optimal solution can be achieved when the subject considers not just self-regarding concerns, but also other-regarding concerns.

The resolution of emotion is observed most acutely at the even-split offer-ratio, because the loss is minimized for both parties. This is consistent with the theory of emotional disparity that sadness is an emotional feedback in the recognition of losses, and the resolution of sadness is to reduce the losses. The greatest reduction of losses to fairness and money in the UG paradigm occurs at the optimal evensplit equal share offer. This phenomenon is also observed for other (happy, angry and jealous) emotions and for fairness perception, which had been reported previously [3-6, 33, 34].

These results together confirmed the hypothesis that the emotional process is an attempt to resolve emotional conflicts as proposed in the emotional disparity theory. Even though most sociologists assert that this equalitarianism is acquired by conforming to social norms for fairness and equality, the relativistic fairness-equity model had also established that this equalitarian state is achieved by optimizing fairness and monetary gains for both parties computationally without any external peer pressure [33, 34].

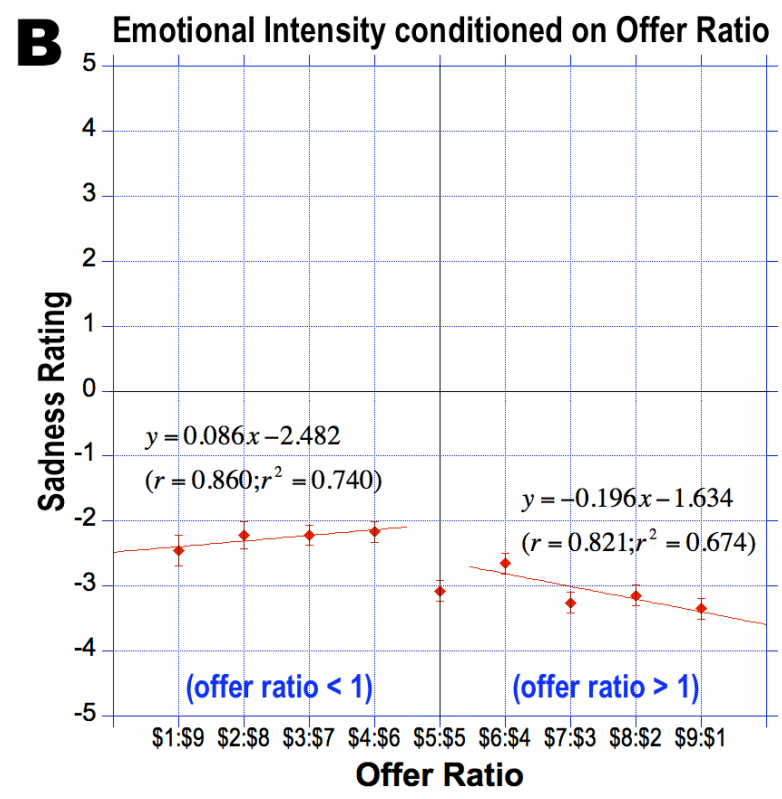

Figure 2. Self-reported sadness rating with respect to monetary offer for acceptance trials only. (A) Curve-fitting to the entire sample showed an inverse proportional relationship. (B) Curve-fitting according to inequitable (unfair) and hyper-equitable (fair) and trials for the same set of data as in (A), which showed different proportionality relationships depending on whether the offers are equitable or not. The error bar represents standard error of mean (SEM).

\subsection{Inter-Relationship between Sadness Level and Decision}

To delineate how sadness and decisions are inter-related, the acceptance trials (Fig. 2) are separated from the rejection trials (Fig. 3) in the analysis. Comparing the stimulusresponse functions for the acceptance trials (Fig. 2A) with the rejection trials (Fig. 3A), it can be observed that there is a $20 \%$ shift in the baseline sadness level ( 2 points in the sadness rating scale of +5 to -5 ). This sadness baseline shift is indicated by the difference in the $y$-intercept of -1.984 (not sad for acceptance decision in Fig. 2A) vs. $y$-intercept of +0.171 (slightly sad for rejection decision in Fig. 3A). It is also obvious that the loss is greater for rejection decision than acceptance decision, because there is monetary gain in accepting the offers, while there is monetary loss in rejecting 
the offers. Thus, this quantitative analysis confirms the hypothesis that sadness is a reflection of the emotional feedback in recognition and resolving the conflicts of losses by accepting the money rather than losing the money in the rejection decision.

Note that the subjects had reported "not feeling sad" (i.e., all data points are below the $x$-axis), when they accepted the offers for all offer-ratios (Fig. 2A). This is consistent with the

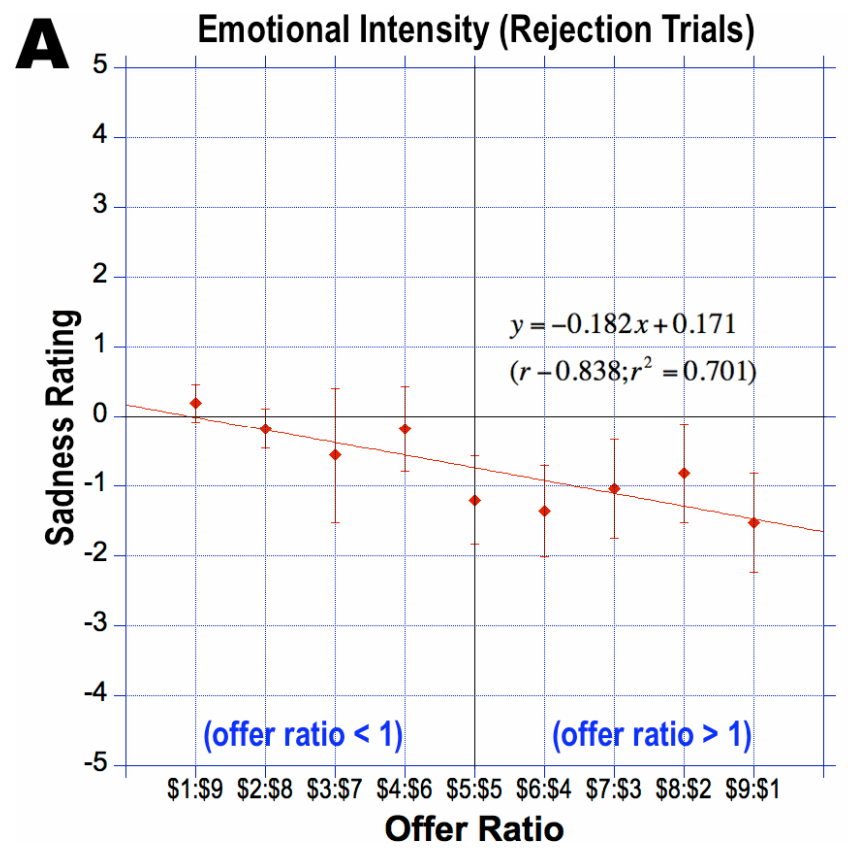

hypothesis that sadness would not be perceived, when they do not lose anything (by accepting the money). On the other hand, if they decided to reject the offers, they only reported slightly sad when the offer was extremely unfavorable (unfair/inequitable) (\$1: $\$ 9$ offer-ratio in Fig. 3B). Nonetheless, the sadness level is related to the disparity in the offer-ratios proportionally, in consistent with the emotional disparity intensity hypothesis.

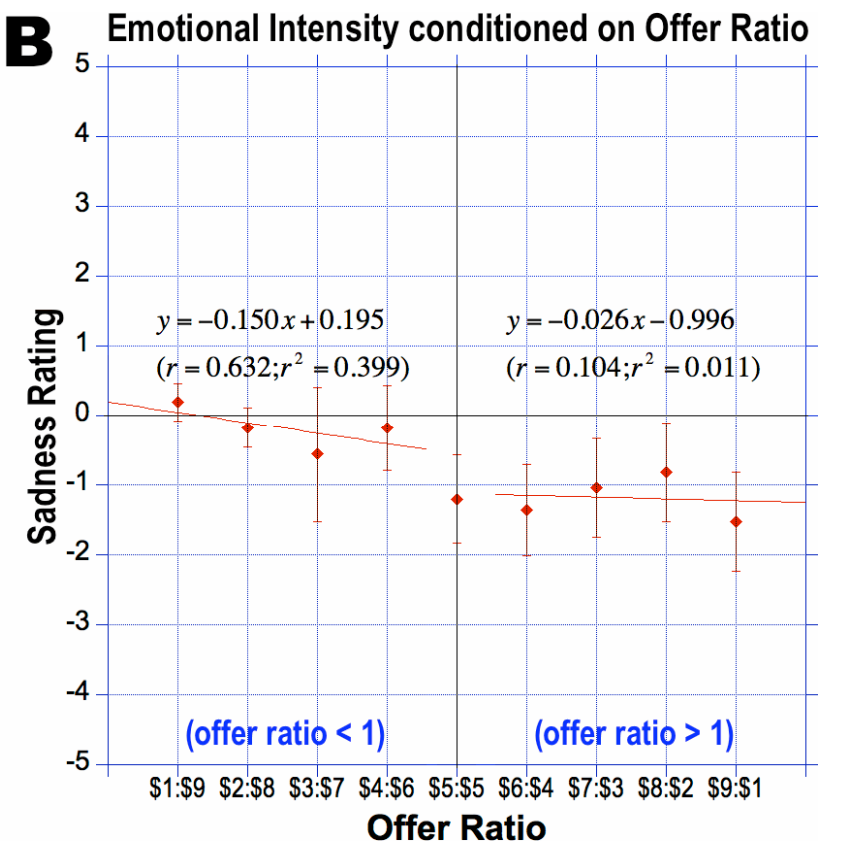

Figure 3. Self-reported sadness rating with respect to monetary offer for rejection trials only. (A) Curve-fitting to the entire sample showed an inverse proportional relationship. (B) Curve-fitting according to inequitable (unfair) and hyper-equitable (fair) and trials for the same set of data as in (A), which showed different proportionality relationships depending on whether the offers are equitable or not. The error bar represents standard error of mean (SEM).

The analysis shows quantitatively how the sad emotion is related to the decision. The sadness perception is related to the decision they made on whether to accept or reject the offer. When they accepted the offers, there is no monetary loss, thus no sadness is felt. On the other hand, when they rejected the offers, there is monetary loss. Yet it is their decision to reject the money, so they are in control of their destiny. Thus, when they have control over the outcome, even though there is a loss, the sad emotion would not be felt. It is only when they have no control over whatever the loss is that sadness sets in. This interpretation is consistent with the sadness hypothesis proposed in this paper.

The slopes are very similar for both decisions, which suggests that the sadness sensitivity is similar for both acceptance and rejection trials (Figs. 2A and 3A), even though there is a baseline shift. The regression coefficients are moderately correlated (regression functions: $y=-0.149 x$ $-1.984 ; r=0.841 ; r^{2}=0.707$ for the acceptance trials in Fig. $2 \mathrm{~A}$ vs. regression function: $y=-0.182 x+0.171 ; r=0.828$; $r^{2}=0.701$ for the rejection trials in Fig. 3A). When the sadness intensities were fitted with a separate curve, conditioned on the hyper-equitable and inequitable offer-ratios (Figs. 2B and 3B), the regressions were not significantly correlated. This shows that the decision to accept or reject only affects the baseline level of sadness intensity, but not the sensitivity to sadness.

\section{Discussion}

The experimental findings validated the hypothesis that the intensity of the sadness level is related to the amount of perceived loss proportionally. The greater the loss, the greater is the level of the sadness intensity. Although most subjects reported not sad to the offers in the UG paradigm, it is consistent with the hypothesis in the emotional disparity theory that sadness is an emotional response to the sense of loss, only when there is no control over the outcomes. Because the subjects do have control over the outcomes (when they played as the responder to the offers in the UG paradigm by making a decision to either accept or reject the money), they would not have felt sad, according to the emotional disparity theory in sad emotional processing.

When the sadness level is compared between the decision to accept and reject the offers, it is found that there is a shift in the baseline level of sadness. The subjects reported less sad for the trials when they accepted the offers than the trials when they rejected the offers. This reduction of sadness level is consistent with the fact that there is less monetary loss when they accepted the offers than when they rejected the offers. This also validates the hypothesis in the sad emotional 
processing for resolving sad emotion by making a decision to accept the offers (which reduces the monetary loss).

These results provided both quantitative and qualitative validation of the hypotheses that sad emotion is proportional to the perceived loss, when there is no control over the outcomes. This interpretation is consistent with the computational process to resolve emotional conflicts by minimizing the loss.

This shows that the decision to alter the outcome can help resolve sadness by reducing the perceived loss. The decision to accept the loss or reduce the loss can be used to bring some congruence between the expected and the actual outcomes. These attempts in resolving the unhappy/sad emotion are all consistent with the emotional processing theory in self-discovery of errors and self-correction of errors to bring the disparity into a congruency. When there is a congruency between the expected and actual outcomes, the unhappy/sad emotion is resolved. The computational process in emotional resolution (as predicted by the emotional disparity theory) provides a means to identify the solutions to reduce the loss, so that a happy/contented state can be achieved. Cognitive awareness of such internal sadness level can provide a person with the effective skills to achieve emotional intelligence by exploring the potential solutions to reduce losses (an incongruent state) in order to achieve happiness (a congruent state).

An example can be used to illustrate one of the effective skills to resolve sadness based on the emotional disparity theory. A relationship breakup can often result in sadness when a person does not want the relationship to end, but realizes that there is nothing he/she can do to save the relationship. To resolve the sadness, a decision can be made to reduce the loss by realizing/accepting that the relationship was not a good match in the first place or that the relationship is not as important as it may seem. One of the solutions to resolve the sadness is to find a better match instead of feeling sorry for the loss. Thus, the effective skill to resolve sadness is the reduction of the perceived loss in emotional intelligence by applying this theory of emotional disparity in real life.

\section{Summary}

The level of sadness intensity is quantified by the stimulus-response function using the UG paradigm, such that a proportionality relationship exists between the sadness intensity and the perceived monetary loss. This confirms that the bigger the disparity between the desirable and the actual outcomes (perceived loss), the greater is the intensity of the sad emotion, especially when the subjects decide to reject the offers. This is consistent with the prediction in the emotional disparity theory that the unhappy emotion (sadness in this case) serves as a feedback for error-correction by assessing the loss. Using the UG paradigm to explore the decisionmaking process, the actual loss can be reduced by the decision to accept the monetary offer instead of rejecting it. Thus, the decision to reduce loss by accepting the money resolves the sad emotion.

By understanding the computational role of sadness in emotional resolution, it provides the motivation to make appropriate decisions to minimize the loss or to accept the loss. The experiment demonstrated that the level of sadness intensity is quantifiable with respect to the disparity between the expected and the actual outcomes. Sadness can be resolved by the decision to reduce the perceived loss. When such an emotional resolution process is brought to awareness, emotional intelligence can be achieved.

\section{Acknowledgement}

I greatly appreciate Ms. Krista Smith for the helpful suggestions and proofreading the manuscript.

\section{References}

[1] D. Tam, "EMOTION-I model: A biologically-based theoretical framework for deriving emotional context of sensation in autonomous control systems," Open Cybern Sys J, vol. 1, pp. 28-46, 2007.

[2] D. Tam, "EMOTION-II model: A theoretical framework for happy emotion as a self-assessment measure indicating the degree-of-fit (congruency) between the expectancy in subjective and objective realities in autonomous control systems," Open Cybern Sys J, vol. 1, pp. 47-60, 2007.

[3] D. N. Tam, "Computation in emotional processing: quantitative confirmation of proportionality hypothesis for angry unhappy emotional intensity to perceived loss," Cogn Comput, vol. 3, pp. 394-415, 2011/06/01 2011.

[4] N. D. Tam, "Quantification of happy emotion: Proportionality relationship to gain/loss," Psychol Behav Sci, vol. 3, pp. 60-67, April 6, 20142014.

[5] N. D. Tam, "Quantification of happy emotion: Dependence on decisions," Psychol Behav Sci, vol. 3, pp. 68-74, April 6, 2014 2014.

[6] N. D. Tam and K. M. Smith, "Cognitive computation of jealous emotion," Psychology and Behavioral Sciences, vol. 3, pp. 1-7, Dec. 31, 20142014.

[7] J. Jaeger, J. C. Borod, and E. Peselow, "Facial expression of positive and negative emotions in patients with unipolar depression,” J Affect Disord, vol. 11, pp. 43-50, Jul-Aug 1986.

[8] F. Schneider, R. C. Gur, R. E. Gur, and L. R. Muenz, "Standardized mood induction with happy and sad facial expressions," Psychiatry Res, vol. 51, pp. 19-31, Jan 1994.

[9] S. Srivastava, H. O. Sharma, and M. K. Mandal, "Mood induction with facial expressions of emotion in patients with generalized anxiety disorder," Depress Anxiety, vol. 18, pp. 144-8, 2003.

[10] I. Laeger, C. Dobel, U. Dannlowski, H. Kugel, D. Grotegerd, J. Kissler, et al., "Amygdala responsiveness to emotional words is modulated by subclinical anxiety and depression," Behav Brain Res, vol. 233, pp. 508-16, Aug 12012.

[11] W. H. Liu, L. Z. Wang, S. H. Zhao, Y. P. Ning, and R. C. Chan, "Anhedonia and emotional word memory in patients with depression," Psychiatry Res, vol. 200, pp. 361-7, Dec 302012. 
[12] M. J. van Tol, L. R. Demenescu, N. J. van der Wee, R. Kortekaas, M. A. N. Marjan, J. A. Boer, et al., "Functional magnetic resonance imaging correlates of emotional word encoding and recognition in depression and anxiety disorders," Biol Psychiatry, vol. 71, pp. 593-602, Apr 12012.

[13] K. M. Harle and A. G. Sanfey, "Incidental sadness biases social economic decisions in the Ultimatum Game," Emotion, vol. 7, pp. 876-881, Nov 2007.

[14] J. von Neumann, O. Morgenstern, and A. Rubinstein, Theory of games and economic behavior. Princeton, NJ: Princeton University Press, 1953.

[15] J. H. Kagel and A. E. Roth, The handbook of experimental economics: Princeton University Press, 1995.

[16] D. A. Braun, P. A. Ortega, and D. M. Wolpert, "Nash equilibria in multi-agent motor interactions," PLoS Comput Biol, vol. 5, p. e1000468, Aug 2009.

[17] K. Sigmund, C. Hauert, and M. A. Nowak, "Reward and punishment," Proc Natl Acad Sci U S A, vol. 98, pp. 1075710762, Sep 112001.

[18] C. Civai, C. Corradi-Dell'Acqua, M. Gamer, and R. I. Rumiati, "Are irrational reactions to unfairness truly emotionallydriven? Dissociated behavioural and emotional responses in the Ultimatum Game task," Cognition, vol. 114, pp. 89-95, Jan 2010.

[19] J. K. Rilling, A. G. Sanfey, J. A. Aronson, L. E. Nystrom, and J. D. Cohen, "The neural correlates of theory of mind within interpersonal interactions," Neuroimage, vol. 22, pp. 16941703, Aug 2004.

[20] A. G. Sanfey, J. K. Rilling, J. A. Aronson, L. E. Nystrom, and J. D. Cohen, "The neural basis of economic decision-making in the Ultimatum Game," Science, vol. 300, pp. 1755-1758, Jun 132003.

[21] A. G. Sanfey, G. Loewenstein, S. M. McClure, and J. D. Cohen, "Neuroeconomics: cross-currents in research on decision-making," Trends Cogn Sci, vol. 10, pp. 108-16, Mar 2006.

[22] P. Smith and A. Silberberg, "Rational maximizing by humans (Homo sapiens) in an ultimatum game," Anim Cogn, vol. 13, pp. 671-7, Jul 2010.

[23] T. Yamagishi, Y. Horita, H. Takagishi, M. Shinada, S. Tanida, and K. S. Cook, "The private rejection of unfair offers and emotional commitment," Proc Natl Acad Sci U S A, vol. 106, pp. 11520-11523, Jul 142009.
[24] A. Bechara, "The role of emotion in decision-making: evidence from neurological patients with orbitofrontal damage," Brain Cogn, vol. 55, pp. 30-40, Jun 2004.

[25] J. D. Greene, L. E. Nystrom, A. D. Engell, J. M. Darley, and J. D. Cohen, "The neural bases of cognitive conflict and control in moral judgment," Neuron, vol. 44, pp. 389-400, Oct 14 2004.

[26] M. M. Pillutla and J. K. Murnighan, "Unfairness, Anger, and Spite: Emotional Rejections of Ultimatum Offers," Org Behav Human Decis Proc, vol. 68, pp. 208-224, 1996.

[27] S. M. McClure, D. I. Laibson, G. Loewenstein, and J. D. Cohen, "Separate neural systems value immediate and delayed monetary rewards," Science, vol. 306, pp. 503-7, Oct 152004.

[28] E. K. Miller and J. D. Cohen, "An integrative theory of prefrontal cortex function," Annu Rev Neurosci, vol. 24, pp. 167-202, 2001.

[29] G. J. Quirk and J. S. Beer, "Prefrontal involvement in the regulation of emotion: convergence of rat and human studies," Curr Opin Neurobiol, vol. 16, pp. 723-7, Dec 2006.

[30] E. T. Rolls, "Brain mechanisms of emotion and decisionmaking,” Int Congress Series, vol. 1291, pp. 3-13, 2006.

[31] M. van't Wout, R. S. Kahn, A. G. Sanfey, and A. Aleman, "Affective state and decision-making in the Ultimatum Game," Exp Brain Res, vol. 169, pp. 564-8, Mar 2006.

[32] D. Tam, "Variables governing emotion and decision-making: human objectivity underlying its subjective perception," BMC Neuroscience, vol. 11, p. P96, Jul 202010.

[33] N. D. Tam, "Quantification of fairness perception by including other-regarding concerns using a relativistic fairness-equity model," Adv in Soc Sci Research J, vol. 1, pp. 159-169, 2014.

[34] N. D. Tam, "Quantification of fairness bias in relation to decisions using a relativistic fairness-equity model," Adv in Soc Sci Research J, vol. 1, pp. 169-178, 2014.

[35] N. D. Tam, "Rational decision-making process choosing fairness over monetary gain as decision criteria," Psychol Behav Sci, in press.

[36] N. D. Tam, "A decision-making phase-space model for fairness assessment," Psychol Behav Sci, in press. 